\title{
Microscopy Characterization of Organic Pigments and Polymer Composite Materials for Xerography Applications.
}

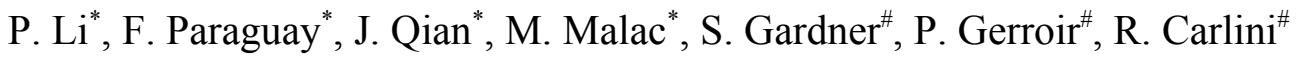 \\ *National Institute for Nanotechnology, Edmonton, Canada. \\ ${ }^{\#}$ Xerox Research Centre Canada, Mississauga, Canada
}

We discuss the characterization of radiation sensitive organic pigment particles by TEM, as well as retrieval of internal microstructure of polymer composite particles used in xerographic toners by electron tomography and by slice-and-view in dual beam (FIB/SEM) instrument.

Printing is a multibillion dollar industry that relies on high-quality marking materials to deliver excellent print quality, speed and value to consumers. New innovations in marking materials require an understanding of the attributes and microstructure of dye or pigments used as colorant. Since the color properties of organic pigments are mainly determined by the choice of chromogen molecule as well as pigment size and morphology[1], then electron microscopy provides a suitable means to investigate the physical structure and microstructure of pigments and other components that are located within toner particles. As both toners and pigment particles are 'soft' organic materials that are highly sensitive to electron beam irradiation, it is necessary to optimize microscopy to maximize information per unit irradiation dose.

We studied commercially available quinacridone-type pigments, such as 2,9-dimethylquinacridone pigment known as Pigment Red 122 (PR 122). We estimated the loss of long range order from loss of intensity in diffraction ring corresponding to $0.3 \mathrm{~nm}$ lattice spacings in the pigment crystallites. At room temperature we have obtained characteristic dose $D_{\mathrm{C}}=(0.35 \pm 0.05) \mathrm{C} / \mathrm{cm}^{2}$. All imaging experiments were performed at less than $1 / 2 D_{\mathrm{C}}$. The dose $D_{\mathrm{C}}$ corresponds to the cross section for carbon $1 \mathrm{~s}$ carbon excitation within a factor of two, suggesting that the primary damage mechanism is due to core loss excitation of carbon [2]. When the samples were observed at $\mathrm{T}=90 \mathrm{~K}$, the characteristic dose increased by about factor of 2.4 . At $\mathrm{T}=90 \mathrm{~K}$, lattice fringes with spacings down to $0.32 \mathrm{~nm}$ were observed in the sample. All experiments were conducted at $200 \mathrm{keV}$ incident electron energy. Fig. 1 shows a typical thickness map obtained at a magnification sufficient to observe $1.6 \mathrm{~nm}$ lattice fringes (the presence and spacing of lattice fringes was confirmed in zero-loss filtered images). Fig. 2 depicts the relationship between the pigment particle external morphology and the crystal lattice planes. Fig. 3 shows a diffraction pattern of the PR 122. The spacings correspond to alpha, beta or gamma structure of 2,9-dimethylquinacridone. The pigment particle shape in 3D was determined from the thickness maps. The distribution of particle sized for PR122 was obtained by examining dimensions of individual particles in the plane perpendicular to the incident electron beam, while the third size dimension of the particle was obtained from thickness mapping by energy-filtering TEM. We have found that the PR122 particles were tetragonal with a long axis $\mathrm{L}=(190 \pm 30) \mathrm{nm}$ while the other two dimensions were found to be $\mathrm{W}=\mathrm{T}=$ $(28 \pm 8) \mathrm{nm}$, an aspect ratio of approximately 7. HRTEM images similar to Fig. 1 revealed that the $1.6 \mathrm{~nm}$ spacing of the molecules is perpendicular to the long axis of the particles, as depicted in Fig. 2. We have often encountered pigment particles where the 
$1.6 \mathrm{~nm}$ lattice fringes were bent confirming that the material can accommodate significant amount of structural stress before the particles break into smaller ones [1].

To study the internal microstructure of polymer composite toner particles, we used electron tomography (ET) [4] and slice-and-view in FIB/SEM. For ET we embedded toner particles in epoxy resin and prepared $80 \mathrm{~nm}$ thick sections using Leica UC6 ultramicrotome. The sections were then stained with ruthenium tetroxide. The ET tilt series were acquired over $\pm 60^{\circ}$ range in JEOL 2200 FS TEM. Fig. 4 shows an example of an image from the tilt series. We have found blade-like domains with long axis $(410 \pm 140) \mathrm{nm}$, width $(100 \pm 20) \mathrm{nm}$ and thickness of about $(39 \pm 3) \mathrm{nm}$ occurring near the surface of the toner particles. The methods described here are being developed to obtain important information about the microstructure of pigment particles and composite toner particles.

\section{References}

[1] Paulus et al. Crystal Engineering Comm. 9 (2007), 131.

[2] H. Qian, M. Malac, R. Egerton, Phil. Mag 87 (2007), 253.

[3] R. Egerton, P. Li, M. Malac, Ultramicroscopy, 35 (2004), 399.

[4] TEMography.com. We acknowledge the extensive help of Dr. H. Furukawa and M. Shimizu on many aspects of TEMography.

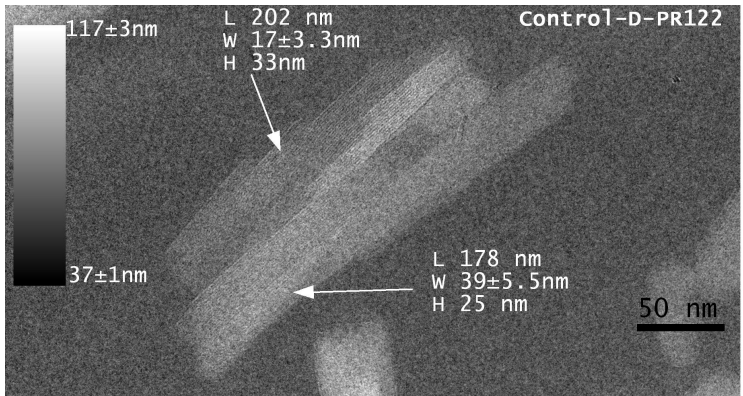

Fig. 1. Thickness map of commercial pigment PR122. The $1.6 \mathrm{~nm}$ lattice fringes can be observed in the top part of the image. Grey scale is calibrated thickness. Scale bar is $50 \mathrm{~nm}$.

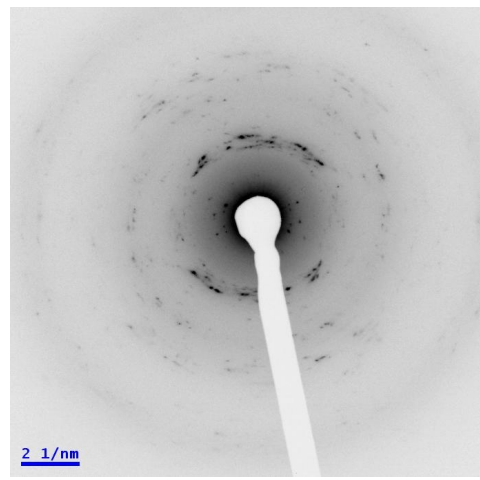

Fig. 3. Diffraction pattern collected at $\mathrm{L}=50 \mathrm{~cm}$ and 200 $\mathrm{keV}$ incident energy.

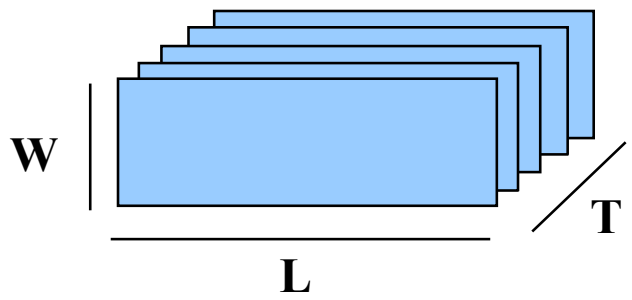

Fig. 2. Schematic picture relating the external shape of PR122 particles to pigment internal structure. The $1.6 \mathrm{~nm}$ lattice planes are perpendicular to the thickness $(T)$ axis.

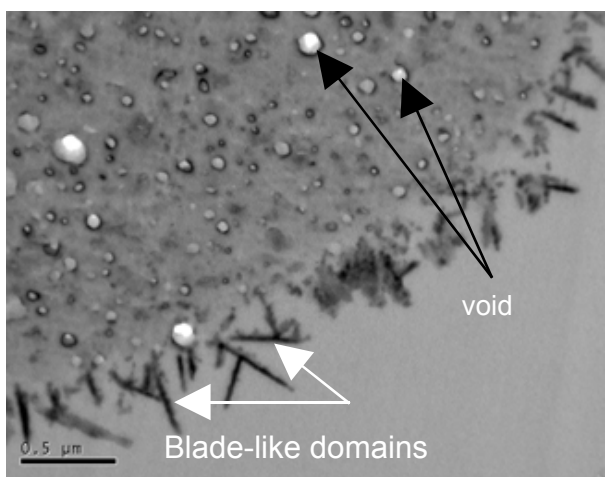

Fig. 4. Projection of electron tomography of toner particle. Blade-like particles marked by an arrow. The scale bar is $500 \mathrm{~nm}$. 